\title{
The MINOS micromegas-TPC Vertex Tracker for ion- beam spectroscopy of very exotic nuclei
}

\author{
Alain Delbart ${ }^{1}$ \\ Irfu, CEA Saclay \\ 91191 Gif sur Yvette cédex, France \\ E-mail: alain.delbartecea.fr
}

\author{
S. Anvar, L. Audirac, G. Authelet, B. Bruyneel, D.Calvet, F. Chateau, A. Corsi, \\ J-M. Gheller, A. Giganon, A. Gillibert, C. Lahonde-Hamdoun, V. Lapoux, \\ D. Leboeuf, D. Loiseau, A. Mohamed, J.-Ph. Mols, A. Obertelli, C. Peron, \\ A. Peyaud, E.C. Pollacco, G. Prono, J-Y. Rousse, and C. Santamaria \\ Irfu, CEA Saclay \\ 91191 Gif sur Yvette cédex, France
}

\begin{abstract}
CEA-Irfu developed a new device, called MINOS (MagIc Numbers Off Stability), to improve both the luminosity and the sensitivity of proton-induced knockout reactions experiments performing in-beam spectroscopy of very exotic nuclei produced at fragmentation facilities (such as RIBF at RIKEN in Japan and in the future at FAIR in Germany). Its innovative approach is that it uses a compact annular $300 \mathrm{~mm}$ long Time Projection Chamber (TPC) surrounding a thick cylindrical liquid hydrogen target. The TPC reconstructs the recoiled proton tracks in order to locate the interaction vertex inside the target, allowing a proper Doppler correction to emitted gamma rays measured in a dedicated detector. A bulk-micromegas, segmented in $4608 \times 2 \mathrm{~mm}^{2}$ pads, is used to readout the TPC which is filled with an Ar$\mathrm{iC}_{4} \mathrm{H}_{10}(3 \%)-\mathrm{CF}_{4}(15 \%)$ gas mixture. The electronics is composed of 20 analog front-end cards. Each of these is read out by a newly designed digital board, called the Feminos, which is compatible with the AFTER and AGET chips. The internal and external TPC field cages are made of $2 \mathrm{~mm}$ thick Rohacell cylinders on which are glued 50 microns thick Kapton foils printed with 381 copper strips. The MINOS device is described, with a focus on the design choices and performances of the TPC characterized in laboratory with cosmic rays. We report on a recent in-beam validation of the TPC at HIMAC (Japan) where a better than $6 \mathrm{~mm}$ FWHM vertex resolution was obtained with a 180 and $350 \mathrm{MeV} / \mathrm{u}^{20} \mathrm{Ne}$ ion-beam impinging on two 0.5 mm thick $\mathrm{CH}_{2}$ targets.
\end{abstract}

Technology and Instrumentation in Particle Physics 2014

2-6 June, 2014

Amsterdam, the Netherlands

\footnotetext{
${ }^{1}$ Speaker
} 


\section{Introduction}

The MINOS project [1] started in October 2010 and is funded by the European Research Council (ERC Starting Grant MINOS-258567) and CEA/Irfu. Its objective is to open a new field of investigations for the study of the shell structure of very exotic and unstable nuclei with an innovative approach for in-beam gamma spectroscopy experiments. The concept (figure 1) is to use a thick liquid hydrogen target (up to $200 \mathrm{~mm}$ long) to greatly improve the luminosity of these experiments and preserve the detection sensitivity and gammas energy resolution by reconstructing the reaction-vertex position in a compact cylindrical Time Project Chamber with a few millimeters resolution. After 3 years of development, the cryogenic target was successfully operated at RIKEN in july 2013. We describe the main relevant characteristics, operating parameters, and performances of the TPC. The TPC performances are finally illustrated by in-beam tests performed at the NIRS-HIMAC facility (Chiba, Japan) in section 3.
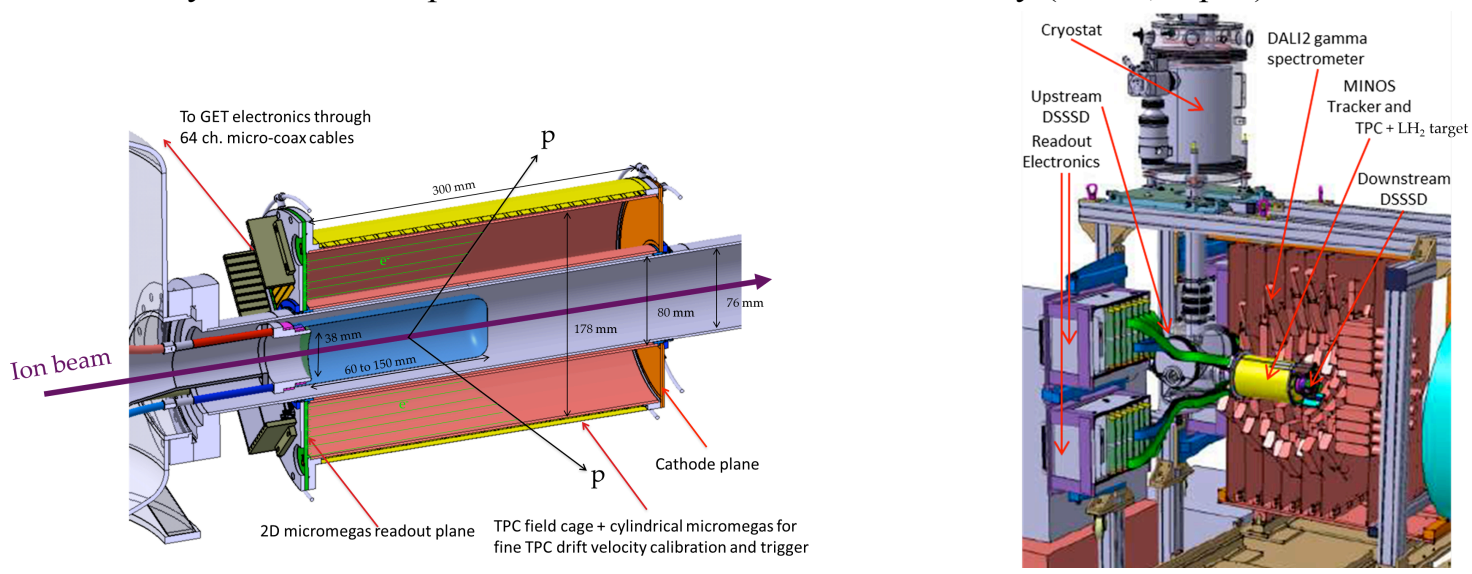

Figure 1: The MINOS apparatus (left) and its integration in the DALI2 [2] gamma ray detector

\section{The MINOS tracker}

Complete Geant4 simulations of the MINOS apparatus show that the sooner the proton are tracked when they exit the aluminum vacuum chamber, the better the resolution is on the vertex position. Using a $128 \mu \mathrm{m}$ amplification gap bulk-micromegas [3], we were able to design and build a very compact light weight TPC field cage described on figure 2 . The TPC walls are made of $2 \mathrm{~mm}$ thick Rohacell on which is glued a $50 \mu \mathrm{m}$ thick Kapton foil (made at CERN/TEMPE-EM). The electric field is defined by $196+195$ one millimeter large strips printed with a $1.5 \mathrm{~mm}$ pitch on both sides of the Kapton foil which has a layout similar to the one of the PANDA TPC [4]. Two 3.9M $\Omega+/-1 \%$ SMD0805 resistors are soldered in parallel between a top and its adjacent bottom strip. The mean value of the resistor between two adjacent top strips was measured to be $3.89 \mathrm{M} \Omega$ for a peak to peak dispersion of $0.25 \%$. The TPC endcaps can be easily dismounted thanks to the solder free connections with the field cage first and last strips respectively located at $4.5 \mathrm{~mm}$ below the cathode plane and $1.5 \mathrm{~mm}$ above the Micromegas mesh. At each step of the TPC vessels assembly, the TPC field cages are electrically controlled 
under $2000 \mathrm{~V}$ for a $2.63 \mu \mathrm{A}$ expected output current. The drift electric field is defined by the applied cathode voltage and the separate voltage applied on the last strip.

The anode plane is segmented in a "projective" way, with 4608 pads organized in 18 concentric $2 \mathrm{~mm}$ large rings of 256 pads (see figure 3). The anode Printed Circuit Boards were manufactured by the ELTOS company (Arrezo, Italy). Twelve internal layers and more than 18000 blind vias are needed to route the pads to four group of nine 160 pins connectors soldered on the backside of the PCB. Low capacitance $(50 \mathrm{pF} / \mathrm{m}) 0.8 \mathrm{~m}$ long Hitachi FC-Band microcoaxial cables were used to transport the pad signals to the Front-End electronic cards.

Benefiting from the CLAS12 barrel detector developments [5], we designed and assembled two shells of curved bulk-micromegas trackers (made at CERN/TE-MPE-EM), with a $92 \mathrm{~mm}$ radius of curvature and segmented in $128 \mathrm{C}$-shaped strips, to be used for TPC electron drift velocity calibration and for a time advanced trigger.
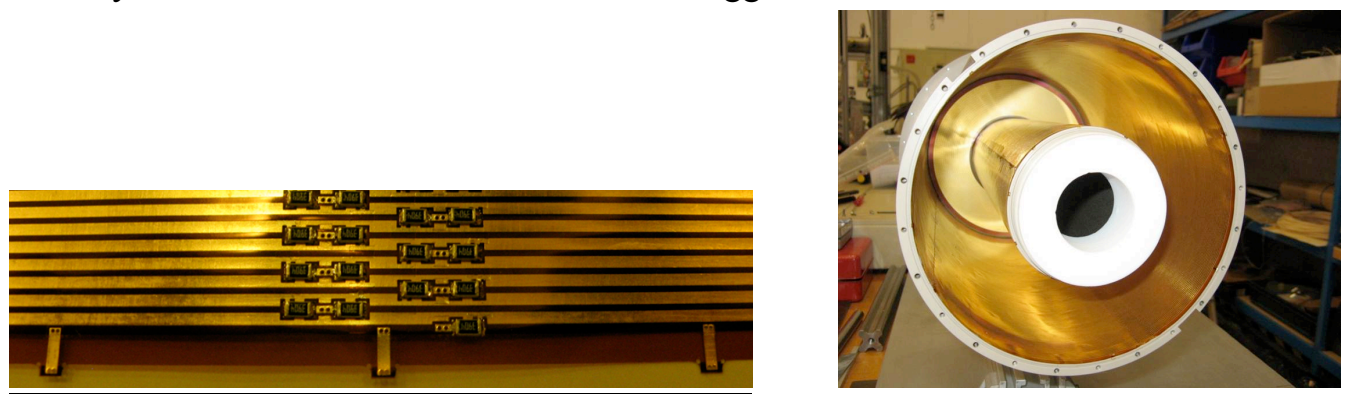

Figure 2: Bottom side of a stripped Kapton foil (left) and picture of the fireld cage (right)

In order to sustain a few $\mathrm{kHz}$ data acquisition rate, the 64 channels AGET chip, designed for the GET project was chosen [6] (General Electronics for TPC, funded by the French funding agency ANR and the DOE (US)). Four AGET chips are mounted on Front-End Cards (FEC) which share the same design as the one used for the readout of the T2K TPCs [3]. Each FEC is connected to a new FEMINOS digital card [7] equipped with an FPGA which performs pedestal subtraction and zero suppression. A new Trigger Clock Module (TCM) was designed to trigger and synchronize up to 24 FEMINOS. The Data Acquisition system "MORDICUS" is based on a $\mathrm{C}++$ generic framework which was developed at Irfu within the GET collaboration. In the operational conditions, the electronic noise was measured to be below 2000 electrons r.m.s.
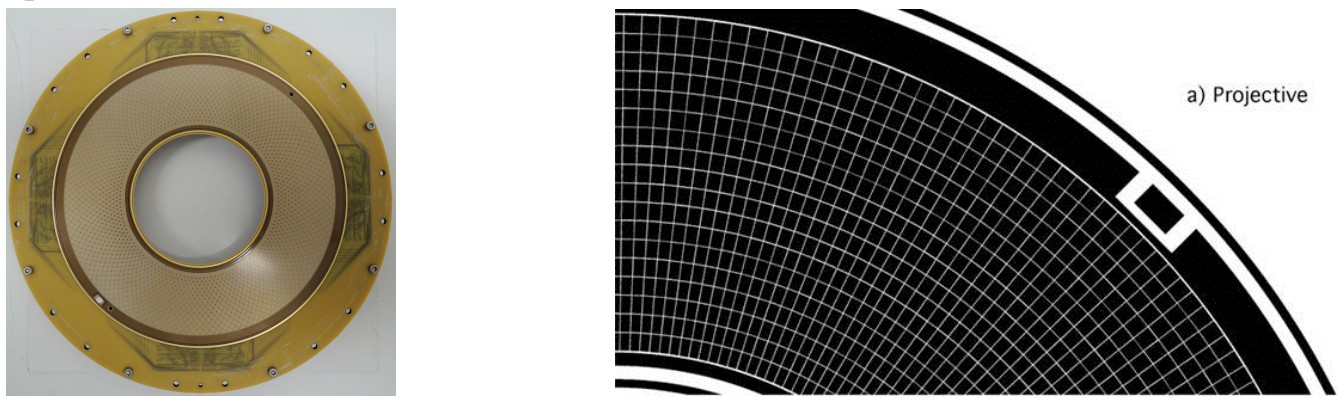

Figure 3: Picture of the bulk-micromegas (left) and anode pad segmentation (right)

The baseline gas mixture is composed of $\operatorname{Ar}(82 \%), \mathrm{CF}_{4}(15 \%)$ and $\mathrm{iC}_{4} \mathrm{H}_{10}(3 \%)$. For a 220 $\mathrm{V} / \mathrm{cm}$ drift electric field the $5.8 \mathrm{~cm} / \mu$ s electron drift velocity matches the $100 \mathrm{MHz}$ sampling frequency of the readout electronics. Both electron transverse and longitudinal diffusions below 
$200 \mu \mathrm{m} / \mathrm{sqrt}(\mathrm{cm})$. The TPC can also be operated without $\mathrm{CF}_{4}$ at $140 \mathrm{~V} / \mathrm{cm}$ (figure 4). The TPC gas leak was measured to be better than $0.1 \mathrm{l} / \mathrm{h}$ and are balanced with a $10 \mathrm{l} / \mathrm{h}$ gas flow to maintain water and oxygen contaminations below monitored $700 \mathrm{ppm}$ and $40 \mathrm{ppm}$ respectively. The operating micromegas gain is set at 2000 for a mesh voltage of $480 \mathrm{~V}$.

\section{In-beam validation of the MINOS Tracker at NIRS-HIMAC (Chiba, Japan)}

The MINOS tracker was validated by a one week beam test campaign performed in October 2013 at the Heavy Ion Medical Accelerator (HIMAC, Japan), in collaboration with collaborators from CNS-The University of Tokyo, NIRS-HIMAC, IPN Orsay, RCNP-Osaka University, RIKEN Nishina Center, and the Tokyo Insitute of Technology. The ${ }^{20} \mathrm{Ne} 350 \mathrm{MeV} / \mathrm{u}$ incident ions were tracked with 2 upstream and downstream MWPCs and were interacting with two $0.5 \mathrm{~mm}$ thick $\mathrm{CH}_{2}$ targets, placed $124 \mathrm{~mm}$ apart in place of the $\mathrm{LH}_{2}$ target. The preliminary results of the the performances [8] of the MINOS TPC are illustrated on figure 4.
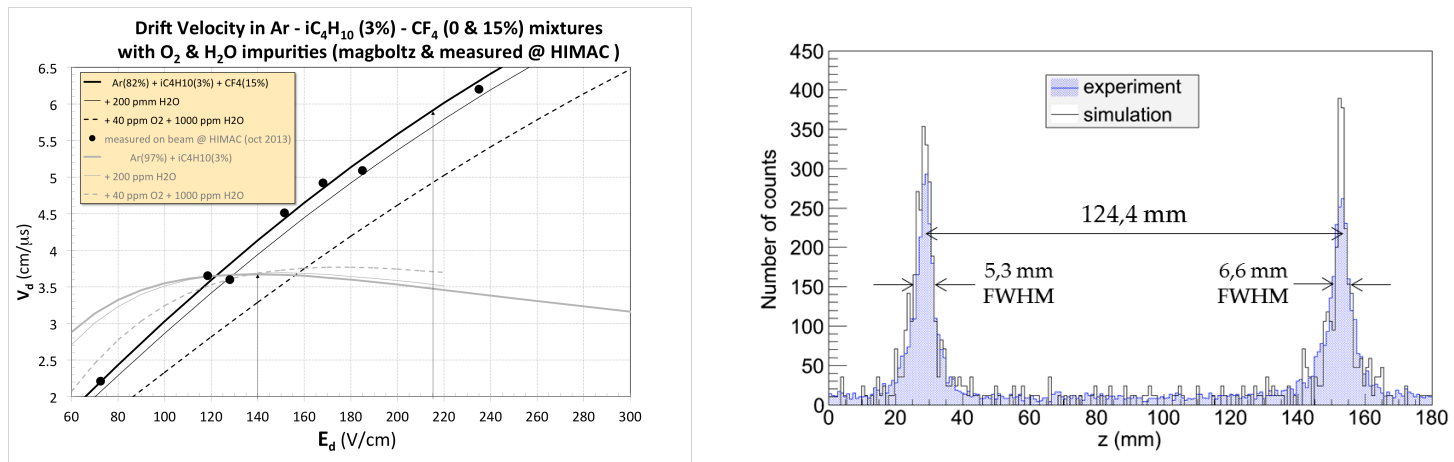

Figure 4: Electron drift velocity (left) and vertex position reconstructed with the TPC

The first physics experiment with MINOS and DALI2 (figure 1) took place at RIKEN/RIBF at the Zero-Degree Spectrometer [9] focal point in May 2014. Results will be described in a coming paper.

\section{References}

[1] A. Obertelli et al., MINOS : A vertex tracker coupled to a thick liquid-hydrogen target for in-beam spectroscopy of exotic nuclei, Eur. Phys. J. A (2014) 50: 8

[2] S. Takeuchi et al., Development of NaI(TI) detector array for in-beam $\gamma$-ray spectroscopy, in RIKEN Accelerator Progress Report (RIKEN, 2005) col. 36, p. 148.

[3] N. Abgrall et al., Time Projection Chambers for the T2K Near Detectors, Nucl. Instrum. Methods A637, 24-46 (2011).

[4] L. Fabbietti et al., The PANDA GEM-based TPC prototype, Nucl. Instrum. Methods A628, 204 (2011).

[5] CLAS12 Technical Design Report, v5.1, Jefferson Lab (2008).

[6] S. Anvar et al., AGET, the GET front-end ASIC, for the readout of the Time Projection Chambers used in nuclear physic experiments, in IEEE Nuclear Science Symposium and Medical Imaging Conference (NSS/MIC) (IEEE, 2011) pp.745-749. 
[7] D. Calvet, A Versatile Readout System for Small to Medium Scale Gaseous and Silicon Detectors, IEEE Trans. Nucl. Sci. 61 (2014) 675.

[8] C. Santamaria et al., Particle tracking with MINOS, to be submitted in Eur. Phys. J.

[9] T. Kubo et al., BigRIPS separator and ZeroDegree spectrometer at RIKEN RI Beam Factory, Prog. Theor. Exp. Phys. 2012, 03C003(2012). 